\title{
Structural, Electrical and Optical Characterization Studies on Glycine Picrate Single Crystal : A Third Order Nonlinear Optical Material
}

\author{
T. Uma Devi *a, N. Lawrence ${ }^{\mathrm{b}}$, R. Ramesh Babu', K. Ramamurthic \\ G. Bhagavannarayana ${ }^{\mathrm{d}}$ \\ ${ }^{a}$ Department of Physics, Government Arts College for Women, Pudukkottaii -622001, India. \\ ${ }^{b}$ Department of Physics, St. Joseph's College (Autonomous), Tiruchirappalli -620002, India. \\ ${ }^{c}$ Crystal Growth and Thin Film Laboratory, School of Physics, Bharathidasan University, \\ Tiruchirappalli-620024, India. \\ ${ }^{d}$ Materials Characterization Division, National Physical Laboratory, Dr. K. S. Krishnan \\ Marg, New Delhi-110 012, India \\ *Corresponding author: kavin_shri@yahoo.co.in \\ Phone: +91 9345123400; Fax: +914312407045
}

\begin{abstract}
Single crystal of an organic nonlinear optical (NLO) material, Glycine picrate (GP), was grown by slow cooling method. The structural perfection of the grown crystal was analyzed by highresolution X-ray diffraction (rocking curve) measurements. UV-Visible-NIR spectral analysis was used to determine the optical constants and band gap of GP. The nature of variation of dielectric constant with frequency at different temperatures was investigated. Third-order optical nonlinearities of GP crystal were investigated. Etch patterns of the grown crystal quality were studied.
\end{abstract}

Keywords: X-ray diffraction, Growth from solutions, Single crystal growth, Nonlinear optic materials

\section{INTRODUCTION}

Organic materials have been demonstrated in recent years to possess superior second and thirdorder NLO properties compared to the more traditional inorganic materials. The properties of organic compounds can be refined using molecular engineering and chemical synthesis. The 
study of amino acid crystallizes with other organic and inorganic molecules, which presents a high non-linear optical coefficient has gained much attention in the last few years because of the possibility of using them in technological devices [1,2]. Our laboratory is engaged in finding new materials for NLO applications and some of these were reported recently [3-6]. Glycine picrate (GP) is an organic nonlinear optical material that belongs to monoclinic crystal system and the lattice parameters are $\mathrm{a}=14.968 \AA, \mathrm{b}=6.722 \AA, \mathrm{c}=15.165 \AA$ and $\beta=93.65^{\circ}$. GP consists of two molecules of glycine and one picric acid molecule and is formed through hydrogen bonding and the carboxyl groups of two glycine molecules share a hydrogen atom [7]. Growth of GP and the results of FTIR, TG-DTA, UV-vis-NIR spectrum and Vicker's microhardness studies of GP were discussed in our earlier report [4].

Third-order nonlinear optical (NLO) materials with weak nonlinear absorption (NLA) but strong nonlinear refraction (NLR) have attracted considerable attention because of their potential uses in the optical signal processing devices [8-13]. The Z-scan method has gained rapid acceptance by the nonlinear optics community as a standard technique for separately determining the nonlinear changes in index and changes in absorption. This acceptance is primarily due to the simplicity of the technique as well as the simplicity of the interpretation.

The dielectric behavior of a material is an important factor as it has direct influence on the NLO efficiency of the crystals. Further quality of the crystal is an important factor when the crystals are brought to the device applications. Hence, we present in this paper, the results of the third order nonlinear optical, dielectric, etching and high resolution X-ray diffraction studies on the nonlinear optical GP crystal.

\section{CRYSTAL GROWTH}

GP was synthesized by the reaction between the organic acid, picric acid (Loba Chemie) and the aminoacid, glycine (Merk) taken in equimolar proportions in aqua solution. The synthesized salt was taken and the saturated solution was prepared in accordance with the solubility data [4]. Slow evaporation of the solvent at room temperature yielded many small crystals. Defect-free, optically clear, and perfectly shaped tiny crystals were chosen as seeds for the growth experiment. Well-developed single crystal of size $10 \mathrm{~mm} \times 9 \mathrm{~mm} \times 5 \mathrm{~mm}$, harvested in a growth period of 7 days is shown in Fig 1. 


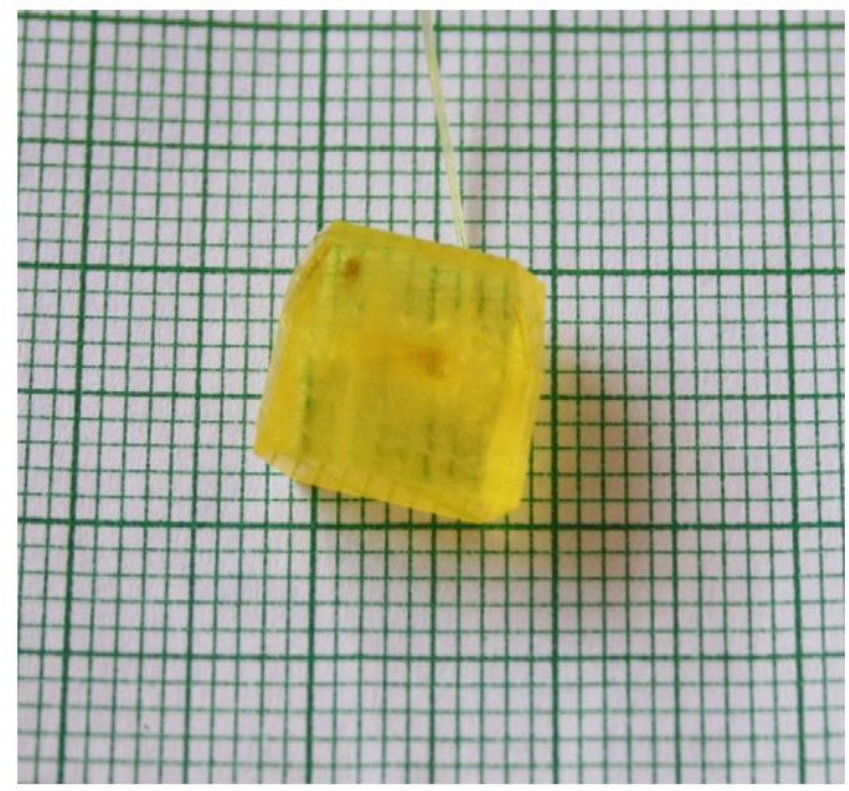

Fig. 1. As grown GP single crystal

\section{EXPERIMENTAL}

\subsection{High-Resolution X-ray Diffractometry Study on GP}

The grown specimen was first lapped and chemically etched in a non preferential etchent of water and acetone mixture in 1:2 volume ratio to remove the non-crystallized solute atoms remained on the surface of the crystal and also to ensure the surface planarity of the specimen. Fig. 2 shows the high-resolution rocking or diffraction curve (DC) recorded for the specimen GP using (002) diffracting planes in symmetrical Bragg geometry by employing the multicrystal Xray diffractometer described above with $\mathrm{MoK} \alpha_{1}$ radiation. As seen in the figure, in addition to the main peak at the centre, this curve contains two more additional peaks. The solid line in these curves which is well fitted with the experimental points is obtained by the Lorentzian fit. The additional peaks at 20 and 40 arc s away from the main peak are due to internal structural very low angle ( $\leq 1 \mathrm{arc} \mathrm{min})$ grain boundaries [14]. The tilt angle i.e. the misorientation angle of the boundary with respect to the main crystalline region for both the observed very low angle boundaries are 20 and 40 arc s. The full width at half maximum (FWHM) values for the main peak and the two low angle boundaries are respectively 26, 16 and 95 arc s. Though the specimen contains very low angle boundaries, the relatively low angular spread of around 5 arc min of the diffraction curve and the low FWHM values show that the crystalline perfection is 
reasonably good. The affect of such low angle boundaries may not be very significant in many applications, but for the phase matching applications, it is better to know these minute details regarding crystalline perfection. It may be mentioned here such very low angle boundaries could be resolved only because of the high-resolution of the multicrystal X-ray diffractometer used in the present investigation.

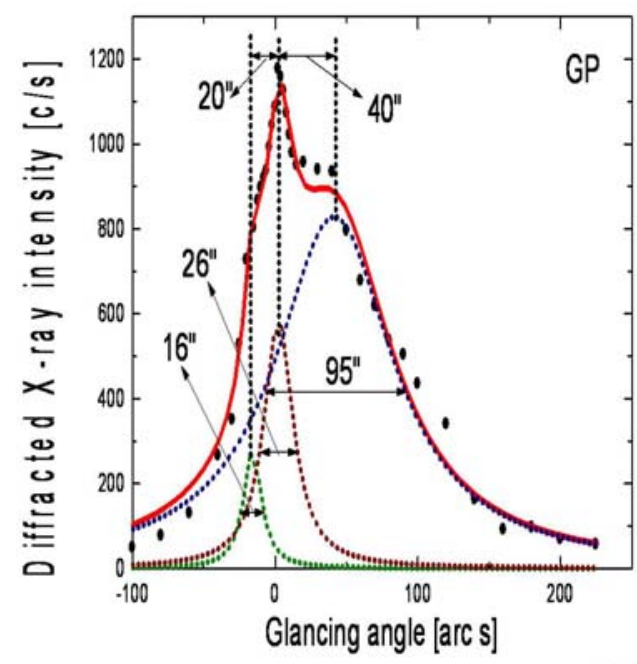

Fig. 2 Diffraction curve recorded for a typical SEST-grown GP single crystal

\subsection{Etching Studies}

The patterns of etch pits depend on the etchant, etching time and crystal faces. Etch pits are associated with dislocations and dislocation bundles and hence bring out the crystal quality [15, 16]. The etch pits in GP crystals were examined using normal incident light type microscope. Etching experiments were performed using water as etchant at room temperature for $10 \mathrm{~s}$ on $(00$ 1) face which was carefully oriented and polished before etching. Fig. 3a shows predominant straight striations on crystals. Some of them extend completely over the surface while others are partly extended. Fig. $3 \mathrm{~b}$ shows the striations parallel to c-axis on its face for etching period of 20 s.

\subsection{Dielectric Studies}

Dielectric measurements were performed on a GP single crystal (dimensions: thickness $1 \mathrm{~mm}$ and area $10 \mathrm{~mm}^{2}$ ) using a LCR meter (Agilent E 4980A) in the frequency range $100 \mathrm{~Hz}-5 \mathrm{MHz}$ and for various temperatures. The variation of dielectric constant with frequency is shown in Fig. 
4. The dielectric constant $\left(\varepsilon^{\prime}\right)$ obtained for GP is higher at the lower frequencies and then it decreases with the increasing frequencies and saturates for further increase in the frequency which can be attributed to space charge polarization mechanism of molecular dipoles [17]. It is important to note that temperature has not influenced much on the dielectric behaviour of the GP crystal. The electronic exchange of a number of ions in the crystals gives local displacement of electrons in the direction of the applied field, which in turn gives rise to polarization. Crystals with high dielectric constant lead to more power dissipation and hence at higher frequencies, the power dissipation in the crystal may have lower value [17].

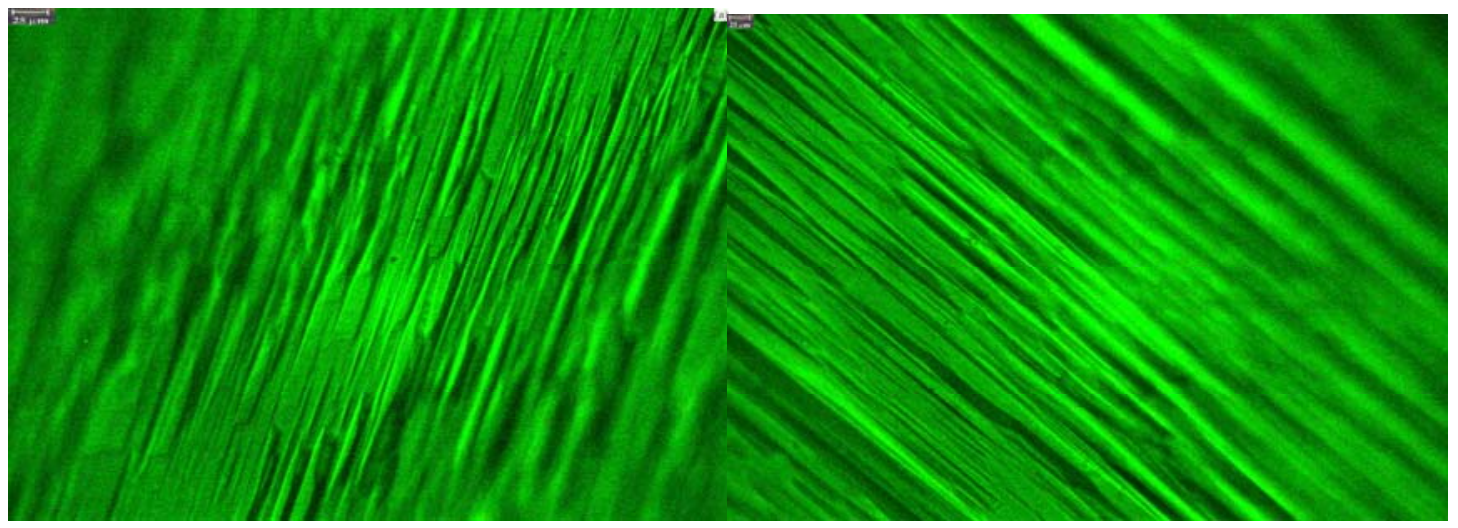

Fig. 3. Etch patterns observed on ( $\left.\begin{array}{lll}0 & 0 & 1\end{array}\right)$ plane of GP single crystal with water as an etchant for a period (a) $10 \mathrm{~s}$ and (b) $20 \mathrm{~s}$

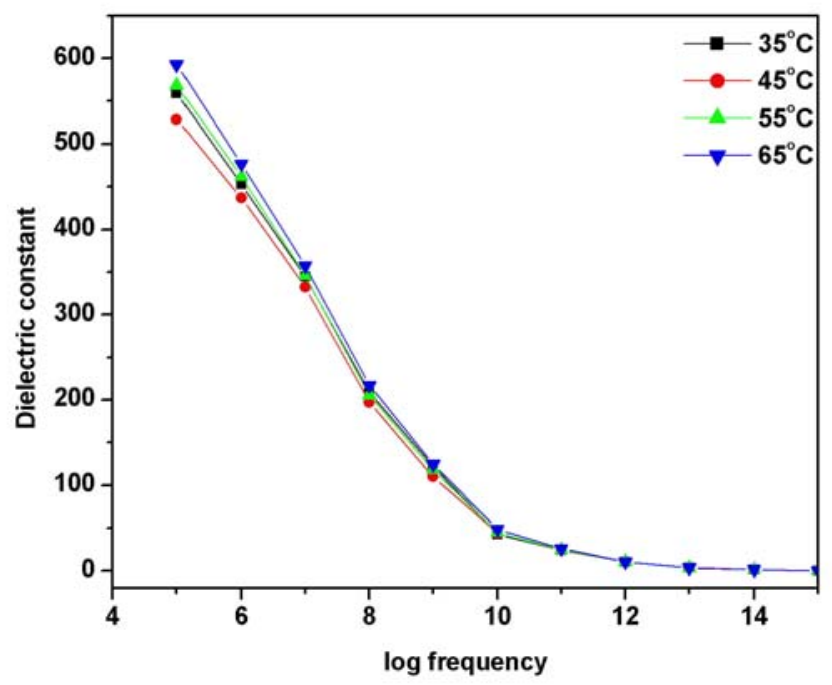

Fig. 4. Dielectric constant vs. log f of GP single crystal 


\subsection{Optical Transmission Studies}

The dependence of optical absorption coefficient with the photon energy helps to study the band structure and the type of transition of electrons [18]. The optical transmittance spectrum of GP recorded using Shimadzu model 1601 in the range 200-1000 nm. Optically clear single crystal of thickness of about $1 \mathrm{~mm}$ was used for this study. There is no appreciable absorption of light in the entire visible range, as is the case for all the amino acids [19]. Some absorption bands in the UVVis region may be attributed to the coloured nature of the material. The short wavelength cutoff occurs at $470 \mathrm{~nm}$. The value of band gap energy was estimated from the graph between hv and $(\alpha h v)^{2}$ by extrapolating the linear portion of the curve to zero absorption (Fig. 5). Here $\alpha$ is the absorption coefficient and hv the photon energy. The band gap energy calculated is about $3.7 \mathrm{eV}$ for the GP single crystal. As a consequence of wide band gap, the crystal under study has relatively larger in the visible region [20]. Values of the refractive index are within the range 2.45 to 2.64. The internal efficiency of the device also depends upon the absorption coefficient. Hence by tailoring the absorption coefficient and tuning the band gap of the material, one can achieve the desired material which is suitable for fabricating various layers of the optoelectronic devices as per the requirements.

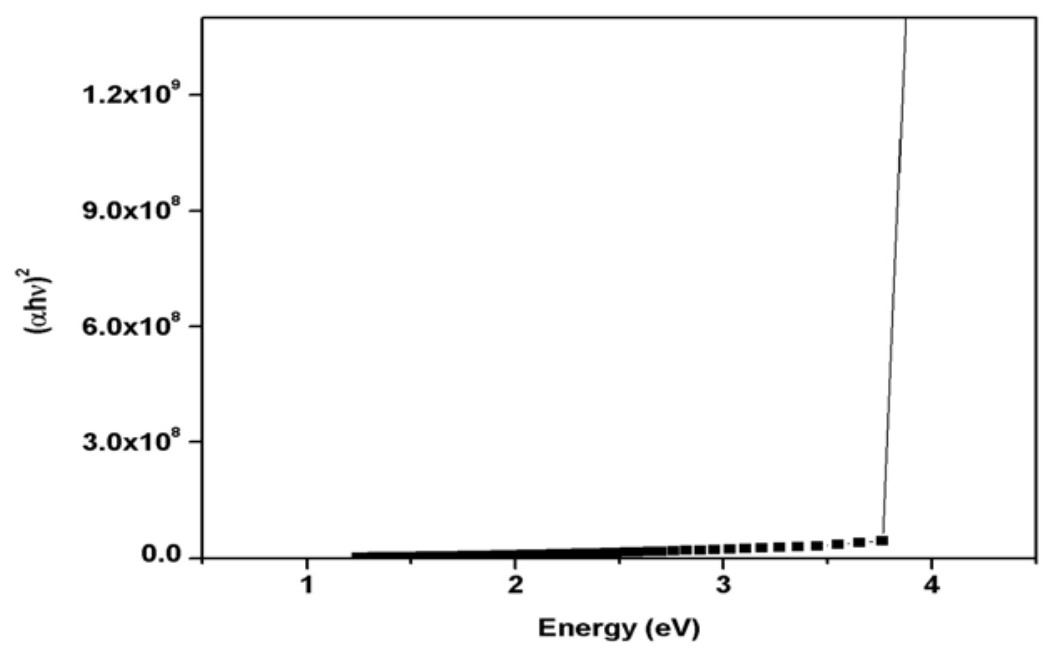

Fig. 5 Graph of hv and $(\alpha h v)^{2}$

\subsection{Z-Scan Measurements}

Third-order nonlinear properties of nonlinear index of refraction and nonlinear absorption coefficient of GP samples were investigated using Z-scan method. Z-scan setup with laser pulses at $632 \mathrm{~nm}$ operating at a $1 \mathrm{kHz}$ repetition rate was employed in this study. The sample thickness used in this experiment was $2 \mathrm{~mm}$. The third-order nonlinear coefficients were estimated by 
measuring the transmittance of a nonlinear medium through a finite aperture in the far field as a function of the sample position $\mathrm{Z}$ with respect to the focal plane. With this procedure the transmittance change between peak and valley $\Delta \mathrm{Tpv}$ can be extracted from them. Using the expression $\Delta \mathrm{T}_{\mathrm{pv}}=0.406(1-\mathrm{S}){ }^{0.25} \Delta \varphi_{\mathrm{NL}}$ we can obtain the induced nonlinear phase, $\Delta \varphi_{\mathrm{NL}}=(2 \pi / \lambda)$ $\mathrm{n}_{2} \mathrm{LI}_{0}$, where $\lambda$ is the wavelength, $\mathrm{L}$ is the sample length, $\mathrm{I}_{0}$ is the intensity at focus and $\mathrm{n}_{2}$ is nonlinear refractive index. Fig. 6 shows Z-scan measurements in the GP crystal. The transmittance change observed as a function of the sample position $\mathrm{Z}$ has a peak to valley shape that is characteristic of a self focusing. GP has a nonlinear refractive index $\mathrm{n}_{2}=4.6 \times 10^{-8} \mathrm{~cm}^{2} / \mathrm{W}$ for the laser wavelength at $632 \mathrm{~nm}$.

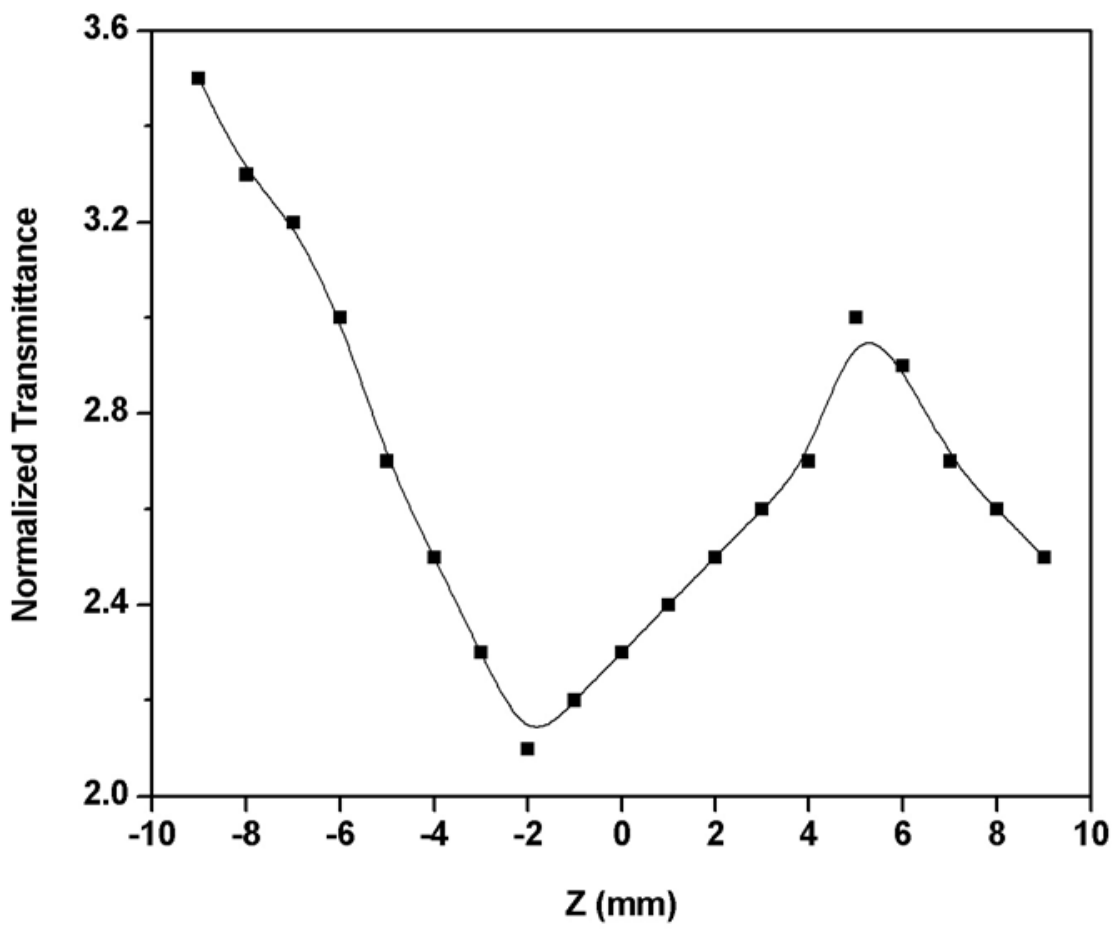

Fig.6. Closed aperture Z-scan measurements on GP crystal

\section{CONCLUSION}

Optically good quality single crystal of GP was grown using temperature reduction technique. A multicrystal high resolution X-ray diffractometer study reveals the crystalline perfection of the crystal without any internal structural grain boundaries. The optical transmittance of the crystal shows the suitability of the crystal for optoelectronic applications. The optical band gap (Eg), absorption coefficient $(\alpha)$ and refractive index (n) were also calculated as a function of energy. Due to the weak inductive effects, the nitro group in picric acid exerts a small shift in the absorption edge of GP around $260 \mathrm{~nm}$ which recommends GP as a better material for the 
fabrication of laser based spectral instruments and also good enough for the generation of higher harmonic light using IR lasers through NLO phenomena. The Z-scan measurements, performed with $632 \mathrm{~nm}$ laser pulses, revealed that GP possesses prominent third order nonlinearity. One can also use this material as third harmonic generator of Nd:YAG laser. Etching studies and the low values of dielectric constant with high frequency for the sample suggest that the sample possesses enhanced optical quality with lesser defects and is a promising material for designing optical device.

\section{REFERENCE}

[1] S. Moitra, T. Kar, J.Cryst. Growth 310(2008) 4539

[2] Z.H. Sun, G.H. Zhang, X.Q. Wang, X.F. Cheng, X.J. Liu, L.Y. Zhu, H.L. Fan, G. Yu, D. Xu, J.Cryst. Growth 310(2008) 2842

[3] T. Uma Devi , N. Lawrence, R. Ramesh Babu, K. Ramamurthi J. Cryst. Growth 310 (2008) 116

[4] T. Uma Devi, N. Lawrence, R. Ramesh Babu, K. Ramamurthi Spectrochim. Acta A 71(2008) 340

[5] T. Uma Devi, N. Lawrence, R. Ramesh Babu, S. Selvanayagam, Helen Stoeckli-Evans, K. Ramamurthi Cryst. Growth Des. 9 (2009) 1370

[6] T. Uma Devi , N. Lawrence, R. Ramesh Babu, K. Ramamurthi Mat. Res. India, 5 (2008) 397

[7] T. Kai, M. Goto, K. Furuhata, H. Takayanagi, Anal. Sci. 10 (1994)359.

[8] J.M. Hales, S.J. Zheng, S. Barlow, S.R. Mrder, J.W. Perry, J. Am.Chem. Soc. 128 (2006) 11362.

[9] S.R. Marder, B. Kippelen, A.K.-Y. Jen, N. Peyhambarian, Nature 388 (1997) 845.

[10] Q. Wang, J. Han, H. Gong, D. Chen, X. Zhao, J. Feng, J. Ren, Adv. Funct. Mater. 16 (2006) 2405.

[11] Y. Zhu, Y.-Z. Zhu, H.-B. Song, J.-Y. Zheng, Z.-B. Liu, J.-G. Tian, Tetrahedron Lett. 48 (2007) 5687.

[12] X. Wang et al., J. Phys. Chem. B 110 (2006) 1566.

[13] M. Sheik-Bahae, A. A. Said, T-H Wei, D. J. Hagan and E. W. Van Stryland, IEEE J. Quantum Electron. 26 (1990) 760.

[14] G. Bhagavannarayana, R.V. Ananthamurthy, G.C. Budakoti, B. Kumar and K.S. Bartwal , J. Appl. Cryst. 38 (2005) 768

[15] J. Bohm, R.B. Heimann, M. Hengst, R. Roewer, J. Schindler, J. Cryst. Growth 204 (1999) 128.

[16] I.H. Jung, K.B. Shim, K.H. Auh, T. Fukuda, Mater. Lett. 46 (2000)354.

[17] L.R. Dalton, J. Phys.: Cond. Matter 15 (2003) 897.

[18] N. Tigau, V. Ciupinaa, G. Prodana, G.I. Rusub, C. Gheorghies, E. Vasilec, Journal of Optoelectronics and Advanced Materials 6(2004) 211 
[19] J.J. Rodrigues Jr., L. Misoguti, F.D. Nunes, C.R. Mendonca, S.C. Zilio, Opt. Mater. 22 (2003) 235.

[20] D.D.O. Eya, A.J. Ekpunobi, C.E. Okeke, Academic Open Internet Journal 17 (2006) 1311. 